\title{
Entre mots et silences
}

L’impossible dialogue de la Célestine

\section{Marie-Blanche Requejo Carrio}

\section{(2) OpenEdition}

\section{Journals}

Édition électronique

URL : http://journals.openedition.org/agedor/924

DOI : 10.4000 /agedor.924

ISSN : 2104-3353

Éditeur

Laboratoire LISAA

Référence électronique

Marie-Blanche Requejo Carrio, «Entre mots et silences », L'Âge d'or [En ligne], 5 | 2012, mis en ligne le

01 mars 2012, consulté le 16 décembre 2019. URL : http://journals.openedition.org/agedor/924 ; DOI :

10.4000/agedor.924

L'Âge d'or. Images dans le monde ibérique et ibéricoaméricain 


\title{
ENTRE MOTS ET SILENCES : L'IMPOSSIBLE DIALOGUE DE LA CÉLESTINE
}

\begin{abstract}
Résumé : Entre théâtre et roman, écrite pour être lue, La Célestine s'écoute telle une polyphonie. Pour chanter les amours de Calixte et de Mélibée, les voix se croisent, s'entrecroisent, se cherchent, s'affrontent et se déchirent pour s'achever sur le chant désespéré d'un père solitaire et déchiré qui reste «triste et seul dans cette vallée de larmes ». Dans cette œuvre singulière, le statut du verbe est fondamental. Conscience tantôt visible tantôt invisible, le verbe y dessine une « image » de la pensée des personnages qui, dans ses différentes modalités d'expression, nous donne à découvrir la vie intérieure des personnages. Nous nous proposons dès lors d'examiner dans cette étude la façon dont les jeux du visible et de l'invisible se manifestent à travers une dialectique de la parole et du silence qui se fait l'écho de la violence des sentiments et des conflits qui animent les personnages. Tout au long de ces jeux, Rojas exprime une scission profonde qui touche l'être humain, seul face à ses passions et à ses blessures, véritable repli sur lui-même qui conduit inéluctablement à la tragédie. Au fil du dialogue et de l'action, nous analysons ces stratégies de l'écriture qui oscille entre voix haute et voix basse, entre ce qui a été dit et ce qui a été marmonné entre les dents. Cette modalité particulière du discours qui échappe à tout paradigme, se révèle au cœur d'un projet d'écriture fondamentalement hybride et permet d'éclairer sous un jour nouveau le problème du genre auquel appartient La Célestine, œuvre qui s'impose à nous comme une tragédie amère reposant sur une vision pessimiste du monde et de la vie.
\end{abstract}

Mots-clés : La Célestine - théâtre - visible/ invisible - violence verbale - secret - rire - tragédie.

Resumen: Entre novela y teatro, escrita para ser leída, La Celestina es una polifonía. Para cantar los amores de Calixto y Melibea, las voces se cruzan y se entrecruzan, se buscan y se enfrentan, murmuran o se callan y todo se acaba con el llanto desesperado de un padre "triste y solo in hac lachrimarum valle". En esta obra el estatuto del verbo es fundamental. Conciencia ora visible ora invisible, el verbo configura una "imagen" del pensamiento de los personajes que, en sus diversas modalidades de expresión, nos ofrece un conocimiento único de la vida interior de los personajes. Nos proponemos por lo tanto examinar en este trabajo, cómo los juegos de lo visible y de lo invisible se manifiestan mediante una dialéctica de la palabra y del silencio que se hace eco de la violencia de los sentimientos y de los conflictos que atañen a los personajes. Por medio de estos juegos, Rojas evidencia una ruptura profunda que afecta al ser humano, solo frente a sus pasiones y sus heridas, verdadero repliegue sobre sí mismo que conduce ineludiblemente a la tragedia. A lo largo del diálogo y de la acción, analizaremos estas estrategias de la escritura que oscila entre una palabra dicha y otra callada, una palabra hablada y otra "murmurada entre dientes". El arte del discurso considerado a esta nueva luz permite enfocar de manera original el problema del género que siempre ha planteado La Celestina que se nos aparece como una amarga tragedia basada en una visión pesimista del mundo y de la vida.

Palabras clave: La Celestina - teatro - visible/ invisible - violencia verbal - secreto - risa tragedia. 
es concepts de visible et d'invisible dans La Célestine donnent lieu à des jeux complexes liés au statut même de l'œuvre entre théâtre et roman. Ecrite pour être lue avant même d'être représentée, la première image que l'œuvre propose à 'auditeur est avant tout une image acoustique, une image sonore que produit ce torrent de mots se déversant de la bouche des personnages. En effet, de même que l'image au sens large est dans l'esprit le signe d'une réalité, le langage, qui lui aussi ressortit au signe, est par excellence le lieu d'une représentation des choses. Les mots, nous rappelle Locke ${ }^{1}$, renvoient aux idées du locuteur dont ils sont les signes mais leur fonction de communication est également essentielle. Les mots, image visible de la pensée sont dits afin d'être clairement entendus. Or, le rapport de force entre les personnages qui caractérise l'ensemble des échanges des acteurs de La Célestine, s'exprime par le biais de stratégies discursives originales. Derrière l'explicite des mots, l'auteur tisse entre les interlocuteurs tout un réseau de rapports implicites qui définissent les lois de la communication dans l'œuvre. En effet, qu'ils soient marmonnés, grommelés, chuchotés, retenus ou interdits, les mots configurent le monde de La Célestine. Afin de mieux blâmer la folie amoureuse et instruire sur la turpitude des serviteurs, Rojas a choisi le dialogue et a conféré aux mots le pouvoir de nous montrer la vie intérieure de ses personnages. Au verbe donc de donner à voir l'indicible.

Car le tourbillon des mots, les échappées du discours, la cacophonie des voix qui se croisent et s'entrecroisent dans La Célestine donnent la mesure de l'esprit de vertige qui y règne. Dès le prologue de la Tragicomédie - édition de Séville de 1502 - le ton est donné. Dans une longue comparatio, Rojas copie un passage du De Remediis de Pétrarque sur le thème de l'universel antagonisme où la succession sans fin d'images de bataille des êtres vivants conduit avant tout à dénoncer la discorde qui agite les hommes. Ce faisant, l'auteur dépeint le spectacle des funèbres soubresauts que traverse une humanité souffrante aux prises avec les effets dévastateurs de la passion. Au-delà d'un exercice de rhétorique, c'est cette prescience qui l'amène à exposer longuement un thème proposé par Héraclite et glosé par Pétrarque. Mais il y appose son style, l'étend à la nature entière et surtout il l'applique à l'homme :

Pues, ¿qué diremos entre los hombres, a quien todo lo sobredicho es subjeto? ¿Quién explanará sus guerras, sus enemistades, sus embidias, sus aceleramientos y movimientos y descontentamientos? ¿Aquel mudar de trajes, aquel derribar y renovar edificios, y otros muchos affectos diversos y variedades que desta nueva flaca humanidad nos proviene ${ }^{2}$ ?

Enfin, ce conflit universel configure naturellement le cadre dans lequel s'inscrivirent les dissensions et les discordes suscitées par le texte de la Comedia : «Y, pues es antigua querella y usitada de largos tiempos, no quiero maravillarme si esta presente obra ha sido

\footnotetext{
${ }^{1}$ «L'homme a des pensées fort diverses et d'autres pourraient comme lui en tirer plaisir et profit ; mais toutes demeurent en son sein, invisibles et cachées aux autres et ne peuvent d'elles-mêmes devenir manifestes. Les avantages et les bénéfices de la vie sociale sont inaccessibles sans communication des pensées ; aussi a-t-il fallu que l'homme trouve des signes sensibles externes permettant de faire connaitre aux autres les idées invisibles dont sont constituées ses pensées. » LOCKE, John, Essai sur l'entendement bumain, Livre III (JeanMichel Vienne, éd.), Paris, Vrin, 2003, p. 39.

${ }^{2}$ Prólogo, p. 199-200. Toutes les références au texte sont empruntées à l'édition de Peter E. Russel. ROJAS, Fernando de, Comedia o tragicomedia de Calisto y Melibea, Madrid, Castalia, 1991.
} 
instrumento de lid o contienda a sus lectores para ponerlos en diferencia, dando cada uno sentencia sobre ella a sabor de su voluntad $»^{3}$.

CEuvre réfractaire à toute tentative d'enfermement dans un sens univoque, La Célestine échappe, dès sa parution, à la transparence du signe qui en garantirait une lecture paisible ${ }^{4}$. Chargées de guérir les plaies d'un lecteur blessé et égaré, les amours de Calixte et de Mélibée trahissent un monde en crise qui a perdu ses valeurs traditionnelles. À l'image de ce monde, fait de violence et de tromperie, les mots vont montrer l'envers du décor, déchirer le voile des apparences pour dénoncer la violence des passions et la confusion du monde. À la suite d'un auteur anonyme, Rojas choisit de relever un défi majeur : construire avec les mots un réel chaotique dans lequel l'être se perd pour finir par se détruire.

L'œuvre entière se construit autour d'un langage qui embrasse la totalité de l'expérience vitale des personnages : le temps (présent vécu, futur espéré, passé), l'espace, les objets, le lieu, les caractères... Au commencement du monde de La Célestine est le verbe. C'est à lui d'exprimer la folie des personnages emportés par leur passion amoureuse, leur cupidité, leur aveuglement. Le langage ne peut plus dire la similitude des choses et perd sa transparence première, celle dont Michel Foucault nous dit qu'elle «a permis la connaissance des choses visibles et invisibles, guidé l'art de les représenter $»^{5}$. Le discours des personnages n'énonce plus la moindre vérité et les lois de la communication s'en trouvent dévoyées. Aveugles au monde qui les entoure, isolés dans un imaginaire qui les condamne à l'avance, les personnages de La Célestine sont des êtres dont le désir signifie la négation de l'autre et de soi.

La confusion du monde s'exprime dans la violence qui caractérise le rapport entre les êtres. Rien de tel dès lors qu'une œuvre dialoguée pour mieux dire l'incommunicabilité qui mène l'être humain à sa perte. Du premier regard de Calixte sur Mélibée à la plainte finale de Pleberio, les voix de La Célestine disent la destruction de l'être pour se confondre en un dernier chant sur la douleur de vivre. La confusion du monde s'y exprime dans la rupture entre le langage et les choses, chargée de dire la tragédie du monde. Entre le silence et la parole, la problématique du discours, placée sous le signe de la subversion, rend compte des choix d'une écriture qui a su explorer de manière originale les voies de la création. Ainsi que Rojas nous y invite dans son prologue, à nous lecteurs de délaisser le «cuento de camino » pour explorer ces "particularidades» qui font de La Célestine une œuvre si singulière. C'est cette problématique que nous nous proposons d'étudier ici6.

\footnotetext{
${ }^{3}$ Ibid, p. 200.

${ }^{4}$ C'est l'épineux problème de l'exemplarité qui se trouve posé là, sur laquelle la critique continue de débattre. Nous ne saurions en proposer ici un état de la question et nous nous contenterons d'évoquer l'ouvrage récent d'Enrique Moreno Castillo qui à notre sens, offre une analyse très claire et convaincante du problème. MORENO CASTILLO, Enrique, La Celestina como tragedia, Sevilla, Iluminaciones Renacimiento, 2010.

${ }^{5}$ FOUCAULT, Michel, Les mots et les choses, Paris, Gallimard, 1966, p. 32.

${ }^{6}$ Évoquant les discordes que suscite la Comedia, Rojas déclare dans son prologue : «Unos les roen los huesos que no tienen virtud, que es la hystoria toda junta, no aprovechándose de las particularidades, haziéndola cuento de camino ». p. 201.

Pour une analyse de ce prologue et des bases d'une "poétique rojasienne », nous renvoyons à l'étude très suggestive de Carlos HEUSCH, «La Littérature selon Fernando de Rojas », Les Langues Néo-latines, $\mathrm{n}^{\circ} 347$, décembre 2008, p. 53-66.
} 
Commençons par la première réplique. «En esto veo Melibea la grandeza de Dios ». Les premiers mots de La Célestine disent l'importance du regard dans une œuvre où le dialogue est tout autant une affaire de regards que de mots. L'on a beaucoup glosé sur cette première réplique de Calixte où la vision du personnage reprend un topique fréquent de la littérature amoureuse du Moyen Age qui fait de la dame aimée une divinité à l'égal de Dieu. Par-delà les références culturelles et littéraires auxquelles il renvoie, ce regard qui ne retient que la beauté de la personne contemplée provoque immédiatement la fureur de Mélibée. L'insulte faite à la jeune fille par un éloge qui la réduit à l'état d'objet désiré7, est le premier faux pas que commet Calixte d'une série désastreuse d'ores et déjà orchestrée par la mauvaise fortune. Car ce que ne voit pas Calixte, c'est la beauté de l'âme, l'ensemble des qualités spirituelles de Mélibée qui devraient être à l'origine de son sentiment amoureux. Dès le début, le regard n'est ainsi envisagé qu'en fonction de ce qu'il ne voit pas, réducteur et agressif, il ne perçoit l'autre que d'une manière partielle, inapte à rendre compte de sa vérité. La première réplique de Calixte, dessine un monde où le réel se dérobe au regard sous le poids des mots. Ceux-là tissent un imaginaire où la vertu et la lasciveté s'opposent, symboles de la violence des relations humaines : «yré como aquél contra quien solamente la adversa fortuna pone su estudio con odio cruel ».

«Esse est percipi aut percipere », «être, c'est être perçu ou percevoir» nous dit Georges Berkeley (1685-1753). C'est en ces termes que le philosophe irlandais rappelle que l'homme ne peut s'appréhender qu'au travers du regard d'autrui, qu'il ne peut « être » que sous son regard et celui du monde. Dévorée des yeux par Calixte et prise sous le feu de sa folle passion, Mélibée n'est qu'une image, image que tour à tour les autres personnages se chargeront de décrire afin de mieux brouiller la vérité d'un être invisible aux autres ${ }^{8}$. Au fil de l'action, le personnage de Mélibée est ainsi appréhendé sous le feu des regards qui se posent sur elle? . Objet de désir et de convoitise, elle va tenter dès les prémices de l'action de faire entendre sa voix et d'opposer à cette image la vérité de son être. Or, sa colère est vouée à l'échec. Il faudra attendre le face-à-face avec Célestine pour entendre de nouveau la voix du personnage. L'on connaît l'issue de la rencontre et la façon dont l'entremetteuse éveille en elle la flamme du désir. La contamination de Célestine empêche toute vérité de se manifester. La philocaptio dont Mélibée est victime en fait à jamais la prisonnière des forces cachées du discours ésotérique ${ }^{10}$. Jusqu'à la fin de l'action, elle sera cette personnalité

\footnotetext{
${ }^{7}$ Voir à ce sujet l'article de June Hall Martin McCash qui, en rappelant le modèle parodié dont fait l'objet le De Amore d'Andreas Capellanus, rappelle que Calixte fait un mauvais usage du manuel d'amour courtois. HALL MARTIN MCCASH, June, "Calisto y la parodia del amante cortés", in Estudios sobre la Celestina (Santiago López Ríos, ed.), Madrid, 2001, p. 475-545.

${ }^{8}$ Les portraits de Mélibée sont nombreux. Calixte sera amené à plusieurs reprises à louer sa perfection puis à évoquer ses coquetteries de femme amoureuse, Célestine évoque l'enfant insignifiante qu'elle a été (VI) Pármeno reconnaît la séduction qu'elle exerce (II et VIII) et Sempronio exprime lui aussi son admiration, laquelle provoque aussitôt la fureur d'Elicia et d'Aréuse qui nous en offrent un portrait beaucoup moins flatteur (IX).

${ }^{9}$ Il s'agit là d'un trait caractéristique de l'écriture qui poursuit la même finalité quel que soit le personnage qui en est l'objet. Ce trait de l'écriture contribue pleinement à l'individuation du personnage et traduit cette propension qu'ils partagent tous à projeter l'autre dans leur imaginaire.

Calixte est ainsi l'objet de plusieurs portraits : de la part de Sempronio (I, II, III, VIII, XI) de Célestine (I, III, IV, VI, IX), de Pármeno (II, VI, VIII, XI), Tristan (XIV), Sosia (XIV), Melibea (IV, XII, XX). Il fait même son acte portrait (XIV). Célestine est décrite par Sempronio (I, III, V, VI, IX, XI, XII), Pármeno (I, II, VIII, XI), Calixte (I, II, VI, XI, XIII), Lucrèce (IV, IX), Alisa (IV, X), Mélibée (IV, XX), Elicia (III, VII), Aréuse (VII, XV) et pratique également l'acte portrait (I, III, IV, V, VII, XI, XII).

${ }^{10}$ Nous entendons par discours ésotérique le processus de construction du personnage de Mélibée dont on ne sait si la passion amoureuse est le fruit des pouvoirs magiques de Célestine où si elle est l'expression d'un sentiment librement exprimé. Le thème de la magie et de la sorcellerie dans La Célestine a fait l'objet de
} 
morcelée, altérée, ayant perdu toute transparence comme peut l'attester l'image naïve que ses parents ont d'elle peu avant la tragédie finale ${ }^{11}$. Le personnage fait ainsi l'objet d'une tension entre deux discours : le discours secret que lui inflige la marque du diable et, au niveau des actions, les marques déchiffrables d'une volonté qui dit son être profond. Elle est dès lors condamnée à n'être ni vue ni voyante. Et c'est bien cette situation qu'exprime le dernier monologue de Mélibée, lorsque seule en haut de sa tour, elle tente d'offrir une dernière vision d'elle-même. La mort arrive à cet instant où le personnage en s'emparant de son être dans un dernier accent de vérité ne pouvait trouver d'autre issue que la mort.

De la même façon, l'absence de regard dénonce la négation de l'autre qu'entraîne l'égoïsme des personnages. Dans l'acte II, Calixte en proie à l'excitation causée par son premier entretien avec Célestine, discute avec Sempronio et le presse de retrouver l'entremetteuse afin de s'assurer qu'elle s'emploie bien à son affaire. Pour ne pas rester seul, il appelle alors Pármeno pourtant présent durant toute la conversation. Quelle gifle reçoit le jeune valet lorsque son maître lui dit avec désinvolture "pues no te veýa » (II, 3, p. 272). Son indifférence est un affront pour celui qui vient de braver Célestine en lui opposant sa loyauté à son maitre. Durant la conversation qui s'ensuit, ce n'est pas la seule réalité physique de Pármeno qui est niée, réalité objective et corporelle, mais son être entier qui est bafoué. Les mots prennent alors le relais du regard pour mieux traduire le mépris dans lequel Calixte, possédé par sa passion amoureuse, tient son valet.

La scène de l'acte II fait apparaître une problématique de la parole surgie des modalités mêmes de l'action. Entre parler et se taire, la pratique langagière des personnages illustre un certain nombre de clichés depuis la nécessité du secret à celle du "buen callar » qui sont autant de manifestations d'une violence verbale attachée à dénoncer l'incommunicabilité et le chaos du monde. Cette dialectique de la parole et du silence est l'un des ressorts du dialogue dans La Célestine et place au cœur de l'action la problématique du secret ${ }^{12}$. Dès le premier acte, l'auteur anonyme a recours à ce ressort dramatique, véritable topique de la littérature courtoise, pour mettre en scène la figure de Célestine et de son monde. La découverte du secret, injure faite à la dame, est en effet la règle allègrement transgressée par Calixte dès lors qu'il se confie à Sempronio. En découvrant son secret, il expose sa passion à la médisance et à la jalousie, figures que vont incarner Célestine et les autres prostituées. À partir de là se développe une casuistique qui confère à l'œuvre toute sa dimension tragique. Sur le plan théologique, la révélation du secret est en effet un véritable péché de langue ${ }^{13}$ dans la mesure où sa révélation porte préjudice à un tiers, en l'occurrence dans le cas qui nous occupe, à l'honneur et à la réputation de Mélibée. Pármeno rappellera cette sagesse proverbiale sur laquelle l'action repose : «a quien dizes el secreto das tu

nombreuses études. Sans prétendre à un état exhaustif de la question, nous invitons à voir à ce sujet les travaux incontournables de Peter E. RUSSEL : "la magia como tema integral de La Celestina » en Temas de "La Celestina » y otros estudios del "Cid" al "Quijote". Barcelona, Ariel, 1978, p. 241-276 ; CÁTEDRA, Pedro M. : Amor y pedagogía en la Edad Media, cap IV : "Amor y magia", Salamanca, Universidad, 1989, p. 85-112 ; VIAN HERRERO, Ana : «El pensamiento mágico en Celestina, "instrumento de lid o contienda" », Celestinesca, 14, 2, 1990, p. 41-91 ; CANET VALLÉS, José Luis, "Hechicería versus Libre albedrío en Celestina », in Lectures de La Célestine, Rennes, Presses Universitaires de Rennes, 2008, p. 61-79.

${ }^{11}$ Cf. l'acte XVI où Lucrèce cachée surprend la conversation des parents de Mélibée et invite la jeune fille à se joindre à elle. Cette image naïve que ses parents ont d'elle provoque une fois de plus la colère de Mélibée. Face à cette image fausse d'elle-même, l'on ne peut que souligner l'étonnant parallèle de ces deux scènes.

${ }^{12}$ La problématique du secret est en effet étroitement liée à la dialectique de la parole et du silence, ainsi qu'il en ressort de la définition même du secret que donne Covarrubias : «todo lo que está encubierto y callado ». Voir à ce sujet la thèse de Michelle GUILLEMONT, Recherche sur la violence verbale en Espagne aux XVI et $X V I I^{e}$ siècles. (Aspects sociaux, culturels et littéraires), Villeneuve d'Ascq, Presses universitaire du Septentrion, p. 15.

${ }^{13}$ Ibid, p. 22. 
libertad $»^{14}$ (II, 3, p. 273). La question du secret se trouve par ailleurs au centre des deux conversations qui voient s'affronter Célestine et Mélibée. Ainsi à l'acte X, c'est autour d'elle que se cristallise toute la violence des deux rencontres où Célestine n'a d'autre volonté que d'obtenir l'aveu si honteux. À la fin de la scène, elle peut savourer avec hypocrisie sa victoire en ces termes : «Y pues así señora, has querido descubrir la gran merced que nos has hecho, declara tu voluntad, echa tus secretos en mi regaço, pon en mis manos el concierto deste concierto » (X, 3, p. 438).

Dans La Célestine, la passion ronge l'esprit qui mène les êtres à se découvrir : "te ruego me dexes hablar un poco, por que aya algún refrigerio » ainsi que Calixte supplie Sempronio afin d'évoquer les charmes ineffables de Mélibée. (I, 4, p. 229) Quant à Mélibée, son évanouissement à l'Acte $\mathrm{X}$, témoigne de la violence de ce désir d'autant plus dévastateur qu'il fait sauter la lourde chape de l'honneur et de la réputation. C'est ce qu'elle exprime dans cette longue tirade où le secret amoureux n'était là que pour préserver les exigences du code. La métaphore érotique de la fleur, si habilement introduite par Célestine a rompu la barrière. La force du désir s'est définitivement emparée de Mélibée qui s'avoue vaincue et découverte :

Quebróse mi honestidad, quebróse mi empacho, afloxó mi mucha vergüença, y, como muy naturales como muy domésticos, no pudieron tan livianamente despedirse de mi cara que no llevasen consigo color por algún poco de espacio, mi fuerça, mi lengua y gran parte de mi sentido. $\mathrm{O}$, pues ya mi nueva maestra, mi fiel secretaria, lo que tú tan abiertamente conoces, en vano trabajo por te lo encubrir (X, 3, p. 437).

Telle est la puissance de Célestine qui donne au corps tout contrôle sur l'esprit et la raison. Tel est l'impact de son pouvoir de perversion qui renverse l'ordre des choses.

Mais dans La Célestine, le comique est inséparable du tragique. Dans la perspective parodique où sont traitées les amours ancillaires, cette thématique du secret se trouve au centre du monologue de Pármeno. À l'acte $\mathrm{X}$, le valet exulte et, loin de songer à cacher quoi que ce soit, il se demande avec qui partager ce plaisir unique : « ¡O alto Dios! ¿A quién contaría yo este gozo, a quién descobriría tan gran secreto, a quién daré parte de mi gloria? ». Après sa nuit d'amour auprès d'Aréuse, le plaisir de Pármeno, pour être total, ne peut être que communiqué. Car, conformément aux leçons de Célestine «el plazer no comunicado no es plazer ${ }^{15}$ (VIII, 2, p. 387). Or, ce besoin de communication ne rencontrera que le rire dégradant et moqueur de Sempronio qui accueille la confidence de Pármeno comme s'il s'agissait là de la reddition du dernier bastion de la raison et la victoire de la folie du monde: "Reyrme querría, sino que no puedo. ¿Ya todos amamos? ¡El mundo se va a perder! Calisto a Melibea, yo a Elicia; tú, de embidia, has buscado con quien perder esse poco de seso que tienes. » (VIII, 3, p. 388). Au plaisir succède l'amertume de celui qui été rudement moqué et rejeté... c'est bien cet échec de la communication que traduit Pármeno par ces mots : «Oýdolo havía dezir, y por esperiencia lo veo, nunca venir

\footnotetext{
${ }^{14}$ Comme le rappellent les éditeurs du texte de la tragicomedia chez Crítica, l'idée très ancienne était présente chez les philosophes présocratiques, et en Espagne apparaissait chez Alphonse le Sage et dans les Proverbios du marquis de Santillana. On retrouve le proverbe cité par Correas « a quien dices tu secreto, das tu libertad y está sujeto » et de nombreux autres qui rappellent la même idée « secreto de dos, sábelo Dios; secreto de tres, toda res». Quant à Covarrubias, il cite un autre proverbe «A uno solo comunica tu corazón, y sea ése muy amigo de el alma », proverbe glosé par saint Cyrille dans ses apologues moraux.

${ }^{15}$ L'idée de l'amitié et du plaisir des joies partagées est un des arguments que Célestine évoque afin de gagner Pármeno à sa cause dès le premier Acte : «El deleyte es con los amigos en las cosas sensuales, y especial en recontar las cosas de amores, y comunicarlas. » (I, 10, p. 262).
} 
plazer sin contraria çoçobra en esta triste vida » (VIII, 3, p. 390). Et si l'acte se scelle sur l'amitié entre les deux valets, la cupidité qui les unit ne saurait tromper le lecteur sur la nature de cette nouvelle relation.

Le conflit amoureux des personnages tel qu'il est développé dans l'œuvre, illustre cette opposition caractéristique de la condition humaine, développée chez saint Augustin et reprise dans la casuistique de saint Grégoire, entre le bonheur de silence et le besoin de parole. Dans La Célestine, la parole est en effet dangereuse, synonyme de tromperie et d'hypocrisie. Celui qui parle se livre, surtout lorsqu'il a pour adversaire Célestine, véritable maîtresse du langage trompeur ${ }^{16}$. À cette parole dangereuse s'oppose le «buen callar», le silence de celui qui sait se montrer prudent ou obéissant. Covarrubias commente longuement la nécessité du silence à propos duquel il écrit «El hablar poco, y el ser los hombres callados, está muy encomendado, así en las letras divinas como en las humanas » et rappelle certains proverbes : «Al buen callar llaman santo ; quien calla piedras apaña [...] en boca cerrada no entra mosca $"{ }^{17}$. Signe de prudence, d'obéissance ou encore de civilité tel qu'il apparait dans certains contes populaires ${ }^{18}$, le silence est une qualité primordiale. Et la seule qui dans la Tragicomédie en fait usage, c'est paradoxalement Célestine toujours si loquace. Car le personnage subvertit toute forme d'autorité et la sagesse proverbiale est, chez elle, régulièrement détournée ${ }^{19}$. Tout comme les mots, le silence doit avant tout servir ses intérêts. C'est ce qu'il ressort de l'occurrence de «buen callar» à l'acte V où Célestine, sortant de chez Mélibée, laisse éclater sa joie et savoure une victoire qu'elle estime due à son savoir-faire. Sa dernière remarque résume sa stratégie: "¿Qué fizieran en tan fuerte estrecho estas nuevas maestras de mi oficio, sino responder algo a Melibea por donde se perdiera quanto yo con buen callar he ganado ?» (V, 1, pp. 328-329). Célestine, prêtresse du verbe, sait qu'il faut en user avec prudence et habileté. À quelle stratégie renvoie ce «buen callar»? À la façon dont Célestine a su maintenir jusqu'au bout le suspense, taisant jusqu'au dernier moment le motif de sa visite? Dans sa turpitude, n'était-ce pas là une manière de laisser Mélibée se consumer par le désir ? Ou encore, ce «buen callar» renvoiet-il à la façon dont elle a su ménager ces moments de demi-murmure afin d'éveiller la curiosité de son interlocutrice ? Renvoie-t-il à la façon dont elle a supporté insultes et menaces pour mieux préparer son mensonge ? Célestine néanmoins ne saura retrouver cette prudence. À l'acte XII, face aux deux valets survoltés venus réclamer leur part du butin, incapable de se taire, aveuglée par son orgueil, elle n'aura de cesse que de les provoquer et trouvera alors la mort

Nombreuses enfin sont les scènes où les personnages s'enjoignent au silence. Ces allégations au silence accompagnent les moments forts du dialogue où la parole de l'autre est vécue comme une agression soit parce qu'elle s'avère dangereuse, soit parce qu'elle s'oppose au désir ou à la volonté du personnage, soit parce qu'elle est devenue outrancière et ridicule.

\footnotetext{
${ }^{16}$ CORREAS, Gonzalo, «Calle por su vida. Calle, no nos oigan, que no soy en la calle; callando el necio es habido por discreto, o parece discreto ...»V Vocabulario de refranes y frases proverbiales (1627), Madrid, Visor Libros, p. 102.

${ }^{17}$ COVARRUBIAS, Sebastián de, Tesoro de la lengua castellana o española, Madrid, Castalia, 1994, p. 239.

${ }^{18}$ Covarrubias rapporte ainsi l'histoire d'un jeune homme fort niais à qui ses parents recommandent de se taire le jour où ils le présentent à sa future belle famille. Incapable de se retenir, le jeune homme prononce un mot qui trahit sa bêtise. Aucun frein ne le retenant plus, il laisse alors libre cours à ses paroles : "Tíos, ya me han conocido, bien puedo hablar », ibid, p. 240.

${ }^{19}$ À propos de la subversión des Autorités dans La Célestine, voir l'article d’Olivier BIAGGINI, " "Sentencia a mi ver digna de perpetua y recordable memoria" : mise en scène et perversion de l'auctoritas dans La Célestine ", in Lectures de la Célestine, Rennes, Presses Universitaires de Rennes, 2008, p. 125-156.
} 
Ainsi, dans l'acte VI, les serviteurs commentent l'habileté de Célestine qui retarde par ses circonlocutions le moment de relater à Calixte son entrevue avec Mélibée. À l'occasion de ces véritables conversations parallèles qu'offrent les apartés, les valets commentent l'attitude de Célestine et sa ruse afin de soutirer un vêtement à leur maître. Ces apartés marquent surtout l'opposition entre les deux valets et éclairent le début de l'évolution de Pármeno pris entre deux sentiments contradictoires : l'envie face à l'adresse de Célestine qui s'apprête à obtenir une récompense qu'elle n'aura pas à partager et la peur que lui inspire pour lui-même et pour Sempronio l'attitude de Calixte ${ }^{20}$. Cette angoisse qui est la sienne, Pármeno ne peut la taire en dépit du risque qu'il court si Calixte vient à l'entendre : «Calla, hombre desesperado, que te matará Calisto si te oye »(VI, 1, p. 336). "Sempronio, cóseme esta boca, que no la puedo sofrir. jencajado ha la saya! »(VI, 1, p. 337). Dans la scène, la parole de Pármeno dit toute l'indignation et la souffrance de celui qui voit se dérouler sous ses yeux une véritable tragédie. Face à Calixte ayant perdu toute mesure et prêt à tout pour voir son désir satisfait, «Madre mía, abrevia tu razón o toma esta espada y mátame » (VI, 1, p. 337), Pármeno observe, «No es mucha su vida; luto havremos de medrar destos amores. » (Acte VI, 1, p. 336-337). Par-delà l'ironie tragique en œuvre dans la scène qu'exprime cette voix distancée de Pármeno, l'échange se fait écho de cette problématique de la parole médisante dans La Célestine : «maldeziente venenoso » (VI, 2, p. 342) nous dit Sempronio de Pármeno, illustrant ce mal de la médisance qui est l'affaire de tous les personnages de $\mathrm{La}$ Célestine qui aiment à mordre leur prochain.

C'est bien dans cette dialectique du silence et de la parole que se situe ce « hablar » ou «murmurar entre dientes» que la critique a si bien commenté et qui est un des traits saillants de l'écriture de La Célestine. Ce procédé dramatique qui émaille de nombreuses scènes, allant jusqu'à donner lieu à de véritables conversations parallèles, oppose entre eux les personnages du bas de l'échelle sociale. Mais il est aussi l'expression de rapports hiérarchiques ambigus entre autorité et complicité ${ }^{21}$. Les uns veulent dire sans dire, les autres entendre sans entendre, savoir et ne pas savoir. Comme le rappelle Covarrubias, la médisance naît essentiellement de l'envie :

Es una plática nacida de envidia, que procura manchar y obscurecer la vida y virtud ajena. Es un mortal veneno de la amistad, como dice San Agustín, "De Amicia", cap. 13, y es oficio de gente vil y baja a quien ninguna vianda sabe bien si no le tocan en vida ajena. San Bernardo, serm I de "Triplici custodia", dice que la lengua maldiciente y murmuradora es pincel del demonio y semejante a la víbora 22 .

Véritable péché de la langue le murmurar des personnages de La Célestine est une insulte. Le personnage ne tente pas de se dissimuler tout à fait, comme si sa voix, dite pour ne pas être entendue, avait paradoxalement une fonction de vraie communication ${ }^{23}$.

\footnotetext{
${ }^{20}$ Dès le premier aparté de la scène, Pármeno s'adresse à voix basse à Sempronio : «No le pierdas palabra, Sempronio, y verás cómo no quiere pedir dinero porque es divisible », et à la réplique suivante, toujours en aparté : " No es mucha su vida; luto havremos de medrar destos amores. », Acte VI, 1, p. 336-337.

${ }^{21}$ Voir à ce propos ce que rappelle Jean-Marc PELORSON, «Pour une réappréciation des apartés dans $L a$ Célestine : de l'artifice théâtral à la vérité d'un langage », in Les Langues néo-latines, 1992, p. 5-20.

22 COVARRUBIAS, op. cit., p. 769-770.

${ }^{23}$ L'aparté a donné lieu à de nombreux commentaires de la part de la critique, dont María Rosa Lida de Malkiel et Marcel Bataillon. Tandis que Maria Rosa Lida de Malkiel mettait l'accent sur l'héritage de la comédie humaniste dans lequel s’inscrit l'aparté, Marcel Bataillon de son côté a insisté sur sa fonction, à ses yeux essentiellement moralisatrice. Nous ne rentrerons pas ici dans ce débat, mais évoquerons la perspective différente prise par Enrique Moreno Castillo qui privilégie l'interprétation de La Célestine comme tragédie. Nous adhérons ici pleinement à cette perspective qui éclaire sous un jour nouveau de nombreux traits
} 
L'insulte proférée est entendue malgré tout, et suscite tantôt la colère tantôt la curiosité de celui qui, se sentant trahi, éprouve le besoin de se rassurer et d'échapper à la profonde solitude qui est la sienne ${ }^{24}$.

Ainsi, dès le premier Acte, dans la scène opposant Sempronio à Calixte, ce procédé que Rojas va systématiser ensuite, éclaire d'une manière singulière la relation entre maître et valet. Il plane en permanence sur les relations entre les personnages cette menace voilée, ce bruit sourd que forment ces mots prononcés entre les dents, ces mots qui sifflent aux oreilles à l'image de ce serpent qui, le premier, a corrompu Eve. Le dialogue noue une relation faite de défiance et de mensonges, de trahison permanente, et ses conséquences seront tragiques. Mais ce qui est remarquable, c'est qu'en dépit de ces manifestations de la duplicité et de la trahison, à aucun moment, le personnage victime du sarcasme (en particulier Calixte et Mélibée), n'en profite pour lever le voile, mettre fin à la discussion et reconnaître son erreur. Il persiste et signe. La seule capable d'interpréter ces apartés comme des insultes et un danger à son autorité, c'est Célestine ${ }^{25}$. Si ces marmonnements et autres grommellements dévoilent la pensée intime du personnage qui parle, ils éclairent tout autant sur la réaction de l'interlocuteur. Celui-ci, bien qu'averti, ne tente pas d'en savoir davantage et se laisse consciemment berner. C'est que le désir l'emporte. La colère n'est que feinte, convention oblige. Qu'importe alors le manque de respect et la trahison, la rapacité la plus mesquine l'emporte. L'aparté révèle la lucidité de tous les acteurs de ce qui n'est qu'un faux jeu de dupes. Ni trompeurs ni trompés, tous mènent un jeu qui doit satisfaire la cupidité des uns, apaiser l'érotisme des autres. Au-delà d'une convention qui veut que l'aparté reste le mode d'expression des personnages vils, il met en lumière la nature profonde des représentants de la noblesse. Ceux-ci partagent avec les personnages du bas de l'échelle la même fausseté, la même hypocrisie. Enfin, si la pratique de l'aparté met sur pied d'égalité les personnages entre eux, elle les réunit également sur le plan des sentiments. La passion de Calixte interdit toute raison de même que le désir de Mélibée l'emporte sur toute sagesse. Tous deux restent sourds aux conseils de ceux qui, dans un premier temps, leur furent fidèles et loyaux.

Mais la médisance ne s'arrête pas aux marmonnements. L'excès de louange en est une, fort prisé des maîtres en particulier. Conformément au principe de réversibilité qu'affichent de nombreux doublets, l'envers de la médisance est l'éloge, et inversement ${ }^{26}$. Les louanges de Calixte et de Mélibée à l'égard de Célestine sont de véritables insultes : l'on songe à l'éloge de Calixte du premier Acte qui suscite la réaction immédiate de Célestine : «Sempronio, ¡De aquéllas vivo yo! Los huesos que yo roý piensa este necio de tu amo de darme a comer ${ }^{27}$ (I, 9, p.251). Dans une perspective plus ouvertement comique, l'accueil enflammé qu'Aréuse réserve à Sosia à l'Acte XVII trahit son intention fallacieuse. Tout aussi rouée que Célestine, elle informe même Elicia de sa stratégie : «... verás quál lo paro

d'écriture du texte. Revenant sur l'aparté, l'auteur écrit : «... el replegamiento del personaje para expresar su propia interioridad adquiere, paradójicamente, una función comunicativa; pues el mismo hecho de hablar entre dientes, haciendo que el otro oiga pero no entienda, es de por sí insultante. », MORENO CASTILLO, Enrique, La Celestina como tragedia, op. cit. p. 98.

${ }^{24}$ Cf. PELORSON, Jean-Marc, op. cit. , p. 13 et ss.

${ }^{25}$ Voir à ce sujet ses réactions face à Pármeno et à Lucrèce.

${ }^{26}$ Cf. Michèle GUILLEMONT, op. cit. p. 45. L'auteur cite un passage tiré de la Silva de varia lección de Pedro Mexía qui dit : «... Fuele preguntado [a Diógenes] una vez que mordedura de animal era la más ponçoñosa. Respondió él: De los animales bravos, la del maldiciente; y de los mansos, la del lisongero [...] ", Pedro MEXÍA, Silva de varia lección, Primera parte, chap. XXVII, p. 400.

${ }^{27}$ Voir également les éloges fallacieux de Mélibée lors de sa seconde rencontre avec Célestine à l'acte X. Et Célestine en use de même. 
lleno de viento de lisonjas, que piense, quando se parta de mí, que es él y otro no. Y sacarle he lo suyo y lo ageno del buche con halagos, como él saca el polvo con la almohaça a los caballos. » (XVII, 2-3, p. 544-545). L'éloge dans La Célestine est mensonge et s'inscrit dans la stratégie des personnages, véritable outil pour parvenir à leurs fins.

Nous touchons là à l'un des traits essentiels de La Célestine, où le langage est envisagé comme ressort dramatique et contribue à caractériser l'action. Le déferlement des mots en de longues tirades ou en de longs monologues, invite à considérer le langage comme une entité à part entière où le signifiant revêt une certaine autonomie par rapport au signifié ${ }^{28}$. Afin de mieux servir leurs intérêts, de mieux s'adonner au plaisir des sens, de justifier leur peur ou leur égoïsme, les personnages prennent appui sur une rhétorique de la persuasion, accumulant sentences, proverbes, et références multiples aux autorités ${ }^{29}$. Leurs intentions malhonnêtes font du langage un outil de tromperie et de mensonge, destiné à manipuler les autres et à se tromper soi-même. Le monologue de Célestine au début de l'acte IV est à ce titre exemplaire. La vieille maquerelle y établit clairement la différence entre ce qui est dit et ce qui est pensé ${ }^{30}$. La parole ne livre jamais la vérité de l'être. Célestine se convainc ellemême et finit par se tromper en prédisant une fin heureuse à une action dont le lecteurspectateur sait d'avance qu'elle sera funeste. L'écart entre une tradition rhétorique persuasive et l'intentionnalité des locuteurs consacre le divorce entre les mots et les choses. L'intérêt, la cupidité, la soif de jouissance qui les anime s'érige en permanence entre euxmêmes et les autres, les empêchant de se connaitre véritablement et interdisant tout discours sincère ${ }^{31}$. Qu'il s'agisse de Sempronio dans son monologue de l'acte I, de Célestine, de Calixte ou de Mélibée, à l'exception du dernier monologue de la jeune fille, les personnages délibèrent sans délibérer, disent sans dire, emportés par le flux d'une parole qui ne vaut que pour elle-même, comme détachée de l'être qui la prononce. Comme l'a rappelé Enrique Moreno Castillo, le langage s'interpose entre la réalité des choses et la personne qui prononce le discours ${ }^{32}$. Cette autonomie du discours dévoile la vacuité de celui qui le prononce, vacuité de l'être prisonnier de lui-même et de ses passions.

Et pourtant, le besoin de communication est essentiel pour des êtres enfermés dans une solitude à laquelle ils tentent tous d'échapper. Jean-Marc Pelorson l'a rappelé, pour qui le dialogue dans $\mathrm{La}$ Célestine est fait de ruptures entre de longues tirades et de brèves réparties, de questions rhétoriques qui restent sans réponse, le refuge dans le monologue, et bien sûr la pratique de l'aparté. Analysant monologues et apartés, il observe comment ceuxci marquent «autant de lignes de fracture et de dysfonctionnements de la communication » ${ }^{33}$. Faussement délibératifs à l'exception du dernier monologue de Mélibée qui annonce la fin de l'œuvre, ils expriment moins le doute du personnage que son besoin impérieux de s'enivrer de mots afin de mieux fuir sa peur ou son désespoir. Que les

\footnotetext{
${ }^{28}$ La tradition rhétorique dans laquelle s'inscrit La Célestine tend à renforcer cette vision du langage comme entité où la production de mots finit par être une fin en soi. Enrique Moreno Castillo analyse avec finesse ces aspects du langage dans son étude, La Celestina como tragedia, op. cit., p. 150-151.

${ }^{29}$ Voir à ce sujet l'étude de Mercedes BLANCO, «Las piezas liminares de La Celestina: un vestíbulo enigmático ", in La Célestine. Comedia o tragicomedia de Calixto y Melibea. Actes du colloque international du 29-30 janvier 1993 (F. Maurizi, éd.), Caen, Presses de l’Université de Caen, 1995, p. 119-144.

${ }^{30}$ Parlant de Sempronio, Célestine révèle «Que, aunque yo he disimulado con él, podría ser que si me sintienssen en estos passos, de parte de Melibea, que no pagasse con pena que menor fuesse que la vida, o muy amenguada quedasse, quando matar me quisiessen, mantenándome, o açotándome muy cruelmente. » (IV, 1, p. 298).

${ }^{31}$ Cf. MORENO CASTILLO, Enrique, La Celestina como tragedia, op. cit. p. 185.

${ }^{32}$ Ibid, p. 189.

${ }^{33}$ PELORSON, Jean-Marc, op. cit. p. 15.
} 
personnages ressassent leur rancœur, tel Pármeno à l'acte VI, ou la rage, telle Mélibée dans sa longue tirade de l'acte XVI après avoir surpris le projet de ses parents de la marier, les monologues sont à l'image d'une œuvre où la parole intarissable se déverse, que la raison à aucun moment ne peut taire. La fièvre des mots emporte les personnages abîmés par leurs blessures profondes. Cette dialectique du silence et de la parole dans La Célestine dit l'incommunicabilité pour mieux dénoncer la violence du monde et la solitude de l'être humain.

Reste le corps, dernier lien qui unit à l'autre et où l'individu connaît ou se connait. Dans cette société sur laquelle pèsent les contraintes les plus aliénantes, quelle que soit l'appartenance sociale du personnage ou son sexe, la seule issue est la chair. D'où l'emprise qu'exerce Célestine sur cette société, levant tabous et interdits. Prêtresse des plaisirs et des sens, Célestine offre la soupape nécessaire qui leur permet d'exister. Et nul ne s'y trompe qui fonce tête baissée dans les rets de la vieille, quitte à y perdre son honneur et sa réputation. Qu'il s'agisse de la nuit d'amour entre Pármeno et Aréuse, des ébats de Calixte et de Mélibée, on reste frappé par la fureur du désir qui s’empare des personnages : «no me trates de tal manera, ten mesura » (VII, 3, p. 379-380) demande Aréuse à son amant novice, tandis que Mélibée supplie «¡Por mi vida, que aunque hable tu lengua quanto quisiere, no obren las manos quanto pueden! ¡Está quedo señor mío! (XIV, 3, p. 501). Dans une perspective comique cette fois, c'est Lucrèce qui donne le ton de la deuxième scène d'amour de l'acte XIX et perd face à Calixte toute mesure au point que Mélibée s'exclame «Déxamele, no me le despedaces, no le trabajes sus miembros con tus pesados abraços. Déxame gozar lo que es mío, no me ocupes mi plazer» (XIX, 3, p. 571). Et le plaisir va crescendo pour culminer dans une métaphore grossière. Ne se retenant plus, Calixte compare sa partenaire à une volaille qu'il s'apprête à déplumer : "Mel : no me destroces ni maltrates como sueles. ¿Qué provecho te trae dañar mis vestiduras? Cal: Señora, el que quiere comer el ave, quita primero las plumas. » (XIX, 3, p. 571). À leur tour, les lecteurs de Rojas qui souhaitaient voir se poursuivre les amours des amants, ont dû être comblés. La violence des sens s'exprime enfin en termes de voyeurisme et d'exhibitionnisme ${ }^{34}$. Toutes ces scènes se déroulent sous l'œil lubrique et jubilatoire de la vieille Célestine $^{35}$, ou encore sous celui d'une Lucrèce envieuse, invitée également à se récréer du spectacle de la défloration de sa maitresse. Tous sacrifient leur honneur, leur réputation, leur fortune et jusqu'à la vie des leurs pour satisfaire cette faim de jouissance. C'est par elle que l'être s'exprime dans toute sa vérité : «No tengo otra lástima sino por el tiempo que perdí de no gozarlo, de no conoscerlo, después que a mí me sé conoscer » nous dit Mélibée à l'acte XVI après avoir entendu le projet de ses parents de la marier.

Dans un monde en crise écrit Juan Goytisolo, «... l'animalité de l'être humain - son exubérance sexuelle - devient le dernier élément qui préserve l’homme de la chosification et lui rend la conscience d'exister par lui-même ${ }^{36}$ ». L'égoïsme forcené des personnages de

\footnotetext{
${ }^{34}$ Car le pendant du voyeurisme c'est l'exhibitionnisme. Voir à ce sujet l'article de Jean-Pierre JARDIN, "Amor dulce, sensualité, érotisme et pornographie dans La Celestina», in Les Langues Néo-Latines, 4, n³47, 2008, p. 5-13.

35 À l'acte IX, le repas que célèbrent les prostituées et leurs amants chez Célestine est une occasion de se repaitre du spectacle des corps enlacés que la vieille maquerelle ne manquera pas.

${ }^{36}$ «En las épocas de fe escasa o nula [...] la llamada « animalidad» del ser humano - su exuberancia sexual se convierte en el único elemento que preserva al individuo de la cosificación y le restituye la conciencia de existir por sí mismo ». Nous traduisons l'extrait. GOYTISOLO, Juan, «La España de Fernando de Rojas », Disidencias, Buenos Aires, Taurus, 1992 (1977 pour la première édition), p. 36. Voir à ce sujet l'analyse de La Célestine que propose avec sa finesse accoutumée Juan Goytisolo à l'occasion de la parution du livre de Stephen Gilman, ibid., p. 17-44.
} 
La Célestine, qu'il s'exprime par l'érotisme ou la cupidité, signifie le chaos d'un monde en pleine mutation ${ }^{37}$, l'incommunicabilité et une désespérante solitude. Cet égoïsme aveugle les conduit à la négation de l'autre et d'eux-mêmes. Après la mort de son amant, le suicide de Mélibée est la seule issue possible. «La présence du Diable sur terre masque-t-elle l'absence de Dieu au ciel de La Célestine? »s'interroge Florence Delay ${ }^{38}$. L'héroïne va vers la mort avec la même détermination égoïste que celle qui la menait vers Calixte. Quant à Calixte, on l'a souvent dit, sa mort est ridicule, comique parce que anti-hérö̈que alors même que c'est le seul moment de l'action où il suit un élan de compassion et se porte au secours de ses jeunes serviteurs. Cette dérogation aux lois internes de la Tragicomédie est la seule cause de sa mort. La déploration finale de Pleberio, la douleur de ce père qui a tout perdu avec sa fille et pour qui la vie n'a plus de sens est le point d'orgue de l'œuvre. Pour la première fois, la parole est vraie où la douleur et le chaos se transforment en expérience vécue dans la conscience d'un personnage ${ }^{39}$. La seule vérité du monde est la douleur.

Le monde que dépeint Rojas dans La Célestine témoigne de ce moment charnière de la Renaissance à la fin du $\mathrm{XV}^{\mathrm{e}}$ siècle, période de crise d'un monde en gestation où après avoir perdu tout repère, chacun cherche sa voie et sa place dans une société qui a perdu toute harmonie. La problématique de la parole, envisagée dans ce travail comme l'expression privilégiée du divorce entre les signes et les choses témoigne de la violence de cette mutation. Tandis que le langage n'exprime plus la vérité des êtres et les enveloppe tous dans la même opacité, le jeu polyphonique des voix qui s'expriment sous la plume de Rojas nous donne à voir un réel, symbole de la vanité de la vie et des choses, de leur vulnérabilité. C'est à Pleberio qu'il appartient de découvrir ce réel et de le lamenter :

$¡ \mathrm{O}$ vida de congoxas llena, de miserias acompañada! ¡O mundo, mundo! [...] Yo pensaba en mis más tierna edad que eras y eran tus hechos regidos por alguna orden; agora, visto el pro y la contra de tus bienandanças, me pareces un laberinto de errores, un desierto espantable, una morada de fieras, [...] río de lágrimas, mar de miserias, trabajo sin provecho, dulce ponçoña, vana esperança, falsa alegría, verdadero dolor. Cévasnos, mundo falso, con el manjar de tus deleytes; al mejor sabor nos descubres el anzuelo; no lo podemos huyr, que nos tiene ya caçadas las voluntades ${ }^{40}$.

À l'image du monde, entre mots et silence, le langage de La Célestine ne dit plus l'heureuse correspondance des signes. La Célestine signe la fin de l'altérité, la fin du mythe de Babel.

\footnotetext{
${ }^{37}$ L'auteur, et avec lui, de nombreux chercheurs, ont rappelé le contexte socio-historique dans lequel $L a$ Célestine a vu le jour. En quelques années, la Péninsule ibérique connaît de nombreuses transformations : unification des couronnes d'Aragon et de Castille par le mariage d'Isabelle et de Ferdinand, création de l'inquisition en 1478, persécution dont fut victime la communauté juive, drame de l'expulsion en 1492, disparition du royaume nasride, découverte du nouveau monde... tous ces changements témoignent des profondes mutations que connaît la société espagnole du dernier quart du XVe siècle. Concernant le contexte socio-historique, nous invitons à consulter les ouvrages suivants : BENNASSAR, Bartolomé, 1492, un monde nouveau?, Paris, Perrin, 1991 ; DEDIEU, Jean-Pierre (éd.), Les deux éveils de l'Espagne, 1492-1992, Paris, CNRS, 1991.

${ }^{38}$ Florence DELAY nous rappelle cette anecdote dans son essai Mon Espagne or et ciel, Paris, Herman éditeurs, 2008.

${ }^{39}$ Voir à ce sujet ce que dit Enrique MORENO CASTILLO, La Celestina como tragedia, op. cit., p. 233 et ss.

${ }^{40}$ Pleberio, XXI, p. 598-599.
} 